\title{
National audit of the management of peritonsillar abscess
}

\section{H M Mehanna, L Al-Bahnasawi, A White}

\begin{tabular}{|c|}
\hline $\begin{array}{l}\text { See end of article for } \\
\text { authors' affiliations }\end{array}$ \\
\hline $\begin{array}{l}\text { Correspondence to: } \\
\text { Mr Hesham Mehanna, } \\
\text { Department of } \\
\text { Otolaryngology and Head } \\
\text { and Neck Surgery, Royal } \\
\text { Alexandra Hospital, } \\
\text { Paisley PA2 9PN, UK; } \\
\text { HeshamMehanna@aol.com }\end{array}$ \\
\hline $\begin{array}{l}\text { Submitted } \\
23 \text { November } 2001 \\
\text { Accepted } \\
27 \text { February } 2002\end{array}$ \\
\hline
\end{tabular}

An anonymous postal survey of 200 consultants was used to audit the current prevalent practices in the management of quinsy in the UK; 101 responded. The findings reveal that on average an otolaryngology department treats 29 cases per year, the vast majority (94\%) on an inpatient basis. The main initial treatment was needle aspiration combined with intravenous antibiotics. Interestingly, those departments treating more than 20 cases a year are more likely to use needle aspiration, while departments in England and Wales use significantly more incision and drainage than those in Scotland. Incision and drainage $(52 \%)$ was the most common form of treatment of non-resolving patients. The median hospital stay was two days.
W ith the introduction of clinical governance, and the requirement for audit of clinical practices, there is a need for the determination of current prevalent practice. This is especially important in conditions where there are several alternative treatments. Firstly, it provides a benchmark, enabling comparison of audit of individual practices with the prevalent practice. Secondly, it allows assessment (including effectiveness and opportunity costs) of new or alternative treatments, and comparison with the existing prevalent treatment. ${ }^{1-3}$ Thirdly, it highlights areas of treatment requiring further research. All this however may be difficult to achieve, as existing practice may not be well defined. ${ }^{4}$

Peritonsillar abscesses or quinsy, is a complication of acute tonsillitis where there is collection of pus between the fibrous capsule of the tonsil and the superior constrictor muscle of the pharynx. It is one of the commonest causes of acute admission to the otorhinolaryngology wards in the UK. ${ }^{5}$ However, the literature describes several different forms of treatment of the condition, with differences in the initial managementnamely aspiration, incision and drainage, or abscess tonsillectomy ${ }^{6-12}$; in the type and route of antibiotic treatment ${ }^{13}{ }^{14}$; as well as in the settings (whether inpatient or outpatient ${ }^{914}{ }^{15}$ ). There appears to be no agreement on the optimal or prevalent treatment for peritonsillar abscesses. ${ }^{56}$

The aim of this survey was to determine the current prevalent practices in the management of quinsy in the UK, thus providing the data required to compare the effectiveness of interventions for peritonsillar abscesses.

\section{METHODS}

A survey was carried out, by postal questionnaire, of 200 (36\%) consultants selected randomly from the full membership list (560 members) of the British Association of OtolaryngologistsHead and Neck Surgeons (BAO-HNS). Responses were anonymous. A total of 101 surgeons replied, representing a $51 \%$ response rate. Questions were asked on the mode of treatment, the setting, the antibiotic route used, and the numbers treated each year. Details of the questions asked are shown in box 1 .

\section{RESULTS}

All the percentages given are those of the total number of respondents (101). Altogether $68 \%$ managed all their patients as inpatients, $26(26 \%)$ managed the majority $(61 \%-99 \%)$ of their patients as inpatients, and only four (4\%) managed less than $60 \%$ as inpatients. No respondents managed all their patients as outpatients (fig 1 ).

The main initial method of treatment was needle aspiration, being performed by 61 (60\%) of surgeons (fig 2). Twenty five $(25 \%)$ performed incision and drainage on initial presentation. Only one ( $1 \%)$ performed abscess tonsillectomy at first presentation. Interestingly, five $(5 \%)$ treated their patients with intravenous antibiotics alone in the first instance.

In addition, at first presentation, all respondents gave their patients antibiotics. Ninety seven (96\%) respondents gave their patients intravenous antibiotics, three $(3 \%)$ gave intramuscular antibiotics, and one surgeon gave oral antibiotics.

If the abscess did not resolve with the initial treatment, the most common subsequent management was incision and drainage (52\%) (fig 2 ). Other forms of subsequent treatment included repeat aspiration (22\%), abscess tonsillectomy $(12 \%)$, and review of antibiotics ( $1 \%)$.

The median length of inpatient stay was two days $(52 \%$ respondents). The length of stay (fig 3) was one day in 5\% of responses, three days in $25 \%$, four days in $3 \%$, five days in $5 \%$, and no responses were given in $11 \%$.

The average annual number of cases seen by an otolaryngology department was 29. Two per cent of respondents worked in departments which treated five or fewer cases per year , 7\% 6-10 cases, $23 \%$ saw $11-20$ cases, $16 \% 21-30$ cases, $11 \% 31-40$ cases, $11 \% 41-50$ cases, and $17 \%$ worked in departments that treated more than 50 cases per year. No information was available for $14 \%$ of replies (fig 4 ).

Analysis by number of patients treated per year (table 1) shows that needle aspiration is used significantly more by consultants who treat more than 20 patients a year than by consultants who treat fewer than 20 patients a year $\left(\chi^{2}\right.$, $\mathrm{p}=0.003)$. On the other hand incision and drainage is used significantly more by consultants who treat fewer than 20 patients a year than by consultants who treat more than 20 patients a year $\left(\chi^{2}, \mathrm{p}=0.001\right)$. No significant differences were observed for any of the other forms of treatment.

Abbreviations: BAO-HNS, British Association of OtolaryngologistsHead and Neck Surgeons; HTBS, Health Technology Board for Scotland 
Box 1: The management of patients with peritonsillar abscess (quinsy)

Firstly some questions about first presentation of this condition

1. Please indicate below approximately the proportion of patients you manage as inpatients and outpatients on first presentation with tonsillar abscess:

(a) Inpatients: none, $1 \%-40 \%, 41 \%-60 \%, 61 \%-99 \%$, all

(b) Outpatients: none, 1\%-40\%, 41\%-60\%, 61\%-99\%, all

2. On first presentation, what method of treatment would you usually use?

- Needle aspiration

- Incision and drainage

- Abscess tonsillectomy

- Other (state)

3. What form of antibiotics do you usually give to patients on

first presentation?

- Intravenous

- Intramuscular

- Oral

- Don't give

4. If you manage any of these patients as inpatients on their first presentation, what is their average length of stay?

(Number of) days OR information not available

5. If you manage any of these patients as outpatients on their first presentation, approximately how many subsequently require an inpatient admission? (Give as \%)

Now a question about your subsequent management of patients with peritonsillar abscess

6 . If the abscess does not resolve with treatment, please indicate how you would manage these patients:

- Repeat needle aspiration

- Incision and drainage

- Abscess tonsillectomy

- Other (state)

Lastly a question about your department

7. Approximately how many cases of peritonsillar abscess are seen by your department as a whole per year?

5 or fewer, 6-10, 11-20, 21-30, 31-40, 41-50, more than 50 , information not available

If you have any comments to make about the treatment of these patients, please make them on the back of this questionnaire

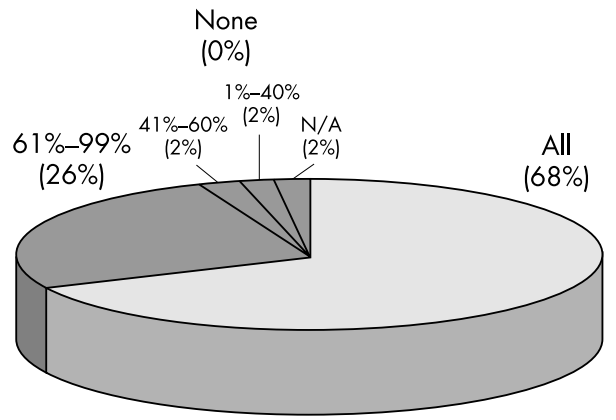

Figure 1 Percentage of patients treated as inpatients $(\%$ of respondents); $N / A$, not available.

Analysis by geography shows that consultants in England and Wales performed significantly more incision and drainage procedures than Scottish based consultants $\left(\chi^{2}, p=0.014\right)$, while the reverse was true for aspiration $\left(\chi^{2}, p=0.012\right)$. No significant differences were found when comparing other forms of treatment.

\section{DISCUSSION}

Health technology assessment is now considered both an ethical and economic imperative. In their duty of care towards

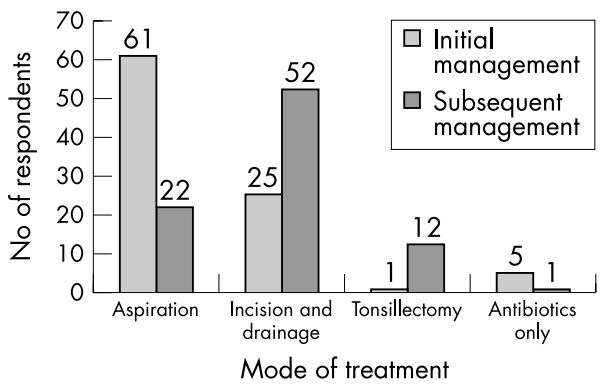

Figure 2 Initial compared with subsequent management.

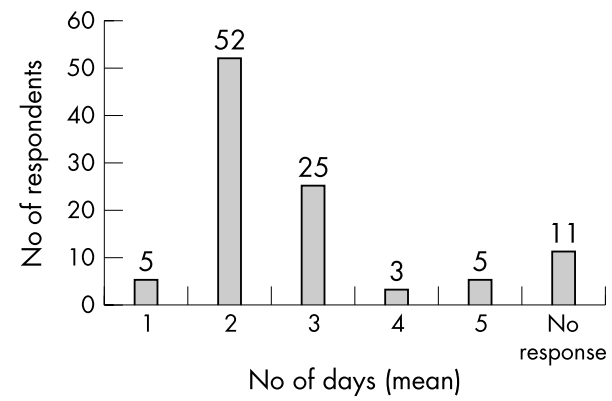

Figure 3 Average duration of inpatient stay.

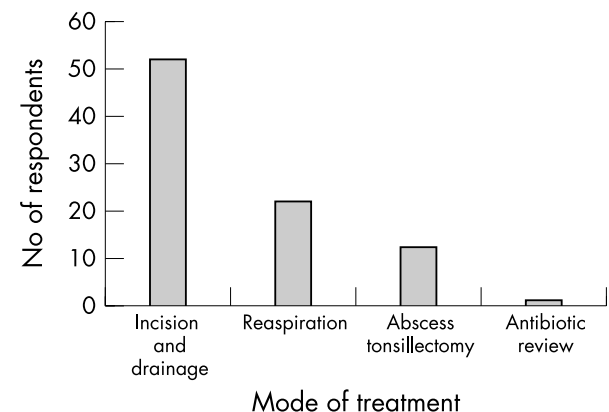

Figure 4 Subsequent management if abscess does not resolve with initial treatment.

Table 1 Analysis of mode of treatment by numbers of patients treated per year

\begin{tabular}{llll}
\hline & $\begin{array}{l}\leqslant 20 \\
\text { Patients/year } \\
(n=31)\end{array}$ & $\begin{array}{l}>20 \\
\text { Patients/year } \\
(n=64)\end{array}$ & $\begin{array}{l}\text { p Value } \\
\left(\text { by } \chi^{2}\right)\end{array}$ \\
\hline A. Needle aspiration & 12 & 45 & $0.003^{*}$ \\
B. Incision and drainage & 14 & 9 & $0.001^{*}$ \\
C. Abscess tonsillectomy & 0 & 1 & 1.00 \\
D. Other & 2 & 3 & 0.66 \\
A + B & 3 & 4 & 0.679 \\
A + D & 0 & 1 & 1.00 \\
B + D & 0 & 1 & 1.00 \\
\hline *Significant at 95\%. & & & \\
\hline
\end{tabular}

patients, clinicians are obliged to ensure that their interventions are clinically effective. Moreover, "since resources are scarce relative to needs, and the use of resources in one way prevents their use in other ways ${ }^{\prime \prime 3}$ there is a need for the assessment and awareness of the cost effectiveness of an intervention. This would allow the elimination of clinically ineffective treatments, and the replacement of less cost effective treatments with more cost effective ones with equal clinical effectiveness. ${ }^{2}$ Moreover, the fact that an intervention has been used for many years is not a guarantee of its effectiveness, clinical or otherwise. ${ }^{16}$ 
The National Institute for Clinical Excellence and the Health Technology Board for Scotland (HTBS) were established "to provide guidance to the NHS on the use of selected new and established technologies"1 and whether "an intervention can be recommended as a cost effective use of NHS resources". ${ }^{1}$ However, they can only assess a limited number of treatments each year; for example the HTBS will assess 10 interventions in 2001-02. ${ }^{17}$ It will therefore fall to the different specialties to perform evaluations of many of the treatments in their field. One such example is the Statements of Clinical effectiveness in Otorhinolaryngology 1998, produced by the BAOHNS. ${ }^{18}$

To be able to assess the effectiveness of different interventions for the same condition, comparison with existing or prevalent practice is essential. ${ }^{2}$ This necessitates that we define and elucidate the prevalent practices, especially for conditions that have several different modes of treatment. It would also seem logical that we concentrate first on the most common conditions or the most expensive treatments, and hence the choice of peritonsillar abscesses which is one of the commonest reasons for acute admissions in otorhinolaryngology.

On a more local level, the introduction of clinical governance necessitates the audit of clinical practices. ${ }^{19}$ Among other things, this necessitates comparison of individual practice with the existing prevalent practice. Thus, the need for the determination of current prevalent practice is further increased, especially in conditions where there are several alternative modes of treatment.

The findings indicate that the majority of surgeons manage patients by needle aspiration, or less commonly by incision and drainage on first presentation. Both these methods have been demonstrated to be equally clinically effective. ${ }^{6-14}$

There are, however, significant differences in the mode of treatment used according to the caseload managed, with departments treating more than 20 patients a year more likely to use aspiration. Moreover there appears to be geographical variations in the choice of mode of treatment, with significantly more surgeons in England and Wales using incision and drainage. The reasons for both these findings are unclear as both treatment procedures require almost exactly the same equipment and facilities to be performed adequately, and require very similar degrees of skill and expertise. In fact, surgeons performing aspiration are usually also trained in incision and drainage in case the abscess recurs. This would therefore suggest that there should not be any significant effects of large caseloads or the type of facilities available on the choice of treatment method. Moreover, as both techniques are equally effective for the treatment of the disease, ${ }^{6-14}$ the severity of the condition when the patient first presents should not be a factor in the choice of treatment method. Hence, in our opinion, variations due to location or number of patients treated cannot be explained on the basis of a more severe caseload, requiring a certain treatment to the exclusion of others. The differences may be due to differences in emphasis during training, or preference of one "school" of technique.

Interestingly, this study demonstrates the fact that almost all surveyed consultants treat the condition on an inpatient basis. This highlights a fundamental difference to the management in the US, where the majority are treated as outpatients. ${ }^{6}$ Moreover, there are several studies that have demonstrated the effectiveness of outpatient treatment. ${ }^{914} 15$ This difference may be due to the differences in set-ups and outpatient support mechanisms of the health services in the two countries, or may be driven by economic factors. It, however, highlights an area that requires further study, and we are currently in the process of evaluating out patient treatment in the UK setting.

Another interesting finding was that all respondents gave intravenous antibiotics after the drainage procedure. There is some evidence however that suggests that oral antibiotics are as effective as parenteral antibiotics, and are more cost effective. ${ }^{13}{ }^{14}$ Further study of mode of antibiotic treatment in peritonsillar abscesses may clarify this issue.

Treatment of the abscess recurrence after initial drainage was found in the survey to be mainly by incision and drainage, although a smaller $(22 \%)$ percentage repeated the needle aspiration, and a few treated it with abscess tonsillectomy. No evidence was found in the literature comparing those methods for treatment of recurrence, highlighting the fact that this has not been studied-probably due to the very low incidence.

\section{CONCLUSION}

Peritonsillar abscess is a relatively common cause of acute admission to an otolaryngology ward in the UK. On average a department treats approximately 30 cases per year. The majority of UK consultants manage patients on an inpatient basis, initially by needle aspiration (61\%) or less commonly by incision and drainage (25\%). All also give them antibiotics, the majority (96\%) intravenously. Interestingly, those departments treating more than 20 cases a year are more likely to use needle aspiration, while departments in England and Wales use significantly more incision and drainage than those in Scotland. For unresolving cases, most (52\%) perform incision and drainage, or less commonly repeat aspiration $(21 \%)$. A small proportion (12\%) resort to abscess tonsillectomy. The median duration of stay was two days. The need was highlighted for further study of treatment of peritonsillar abscesses on an outpatient basis, the optimal mode of antibiotic treatment, and the management of abscess recurrence.

\section{ACKNOWLEDGEMENTS}

We would like to thank the staff of the Departments of Clinical Audit and Clinical Effectiveness at the Royal Alexandra Hospital for the invaluable help they extended to us during this project. We would also like to thank the Scottish Otolaryngological Society for funding this project.

\section{Authors' affiliations}

H M Mehanna, L Al-Bahnasawi, A White, Department of

Otolaryngology and Head and Neck Surgery, Royal Alexandra Hospital, Paisley

\section{REFERENCES}

1 Sculpher M, Drummond M, O'Brien B. Effectiveness, efficiency, and NICE. BM 2001;322:943-4.

2 Russell LB. Opportunity costs in modern medicine. Health Affairs 1992;11:162-9.

3 Gold MR, Siegel JR, Russell LB, et al. Cost effectiveness in health and medicine. New York: Oxford University Press, 1996.

4 Palmer S, Rafferty J. Economic notes: opportunity cost. BM 1999;318:1551-2.

5 Hall SF. Peritonsillar abscess: the treatment options. J Otol 1990; 19:226-9.

6 Herzon FS. Peritonsillar abscess: incidence, current management practices, and a proposal for treatment guidelines. Laryngoscope 1995; 105: 1-17.

7 Wolf $\boldsymbol{M}$, Even-Chen I, Kronenberg J. Peritonsillar abscess: repeated needle aspiration versus incision and drainage. Ann Otol Rhinol Laryngol 1994; 103:554 -7.

8 Epperly TD, Wood TC. New trends in the management of peritonsillar abscess. Am Fam Phys 1990;42:102-12.

9 Herzon FS. Permucosal needle drainage of peritonsillar abscesses. A five year experience. Arch Otol 1984;1 10:104-5

10 Chowdhury CR, Bricknell MC. The management of quinsy-a prospective study. J Laryng Otol 1992;106:986-8.

11 Fagan JJ, Wormald PJ. Quinsy tonsillectomy or interval tonsillectomy-a prospective randomised trial. S Afr Med J 1994;84:689-90.

12 Spires JR, Owens JJ, Woodson GE, et al. Treatment of peritonsillar abscess. A prospective study of aspiration vs incision and drainage. Arch Otolaryngol Head Neck Surg 1987; 113:984-6.

13 Sexton DG, Babin RW. Peritonsillar abscess: a comparison of a conservative and a more aggressive management protocol. Int J Pediatr Otorhinolaryngol 1987; 14:129-32.

14 Stringer SP, Schaefer SD, Close LG. A randomised trial for outpatient management of peritonsillar abscess. Arch Otolaryngol Head Neck Surg $1988 ; 114: 296-8$. 
15 Maharai D, Rajah V, Hemsley S. Management of peritonsillar abscess. J Laryngol Otol 1991;105:743-5.

16 Chalmers I. Invalid health information is potentially lethal. BM 2001322:998

17 Health Technology Board for Scotland (2001). Topics for evaluation (http://www.htbs.org.uk). Accessed 23 March 2001.
18 British Association of Otorhinolaryngologists-Head and Neck Surgeons. Statements of clinical effectiveness in otorhinolaryngology 1998. London: Royal College of Surgeons of England, 1998.

19 British Association of Otolaryngologists-Head and Neck Surgeons. Clinical governance and the role of the BAO-HNS. London: Royal College of Surgeons of England, 2000: 2, 8-9.

FILLER Sources of inspiration

M

most inspirational textbook was the 6th edition of Harrison's Principles of Internal Medicine, strangely enough, not because I read it in preparation for the membership, but because, years afterwards, I came to appreciate more fully the significance of its dedication "to all those who have taught us, and especially to our younger colleagues who continue to teach and inspire us". Starting with our younger colleagues, I can only say "Thank you for all those searching challenges which have helped to keep me on my toes". Some of those challenges raised such fundamental issues that they were recycled as hypotheses worthy to be tested through the medium of a different kind of textbook, namely, the literature search, or through the medium of a fully fledged research project.

With regard to my more formal instruction in medicine, my most inspirational role model was my Professor of Medicine, Barry Adams, who taught me everything I shall ever need to know about that magical synthesis of experiential learning and published knowledge. He had up to date facts and figures of all the common medical conditions diagnosed in patients who had passed through his department, and was capable of raising any clinical discussion to a higher intellectual level by placing experiential knowledge of this kind in the context of published evidence. He also had the honesty to be "up front" with the limits of his knowledge, and to caution against slavish acceptance of conventional wisdom including his own. With his mischievous sense of humour, he relished the idea of "standing the logic on its head", which was one way of searching for new insights and testing new hypotheses. Although he did not quite put it that way, his perspective was that "there were a lot of answers out there, all waiting for the right questions". Above all however, I admired his compassion and his unfailing courtesy towards his patients, many of whom were untutored peasants. Through his example I still inwardly wince when I encounter instances of gratuitously patronising behaviour towards patients. $-\mathrm{O}$ M P Jolobe, Geriatrician, Department of Adult Medicine, Tameside General Hospital, Ashton under Lyne, UK 